\title{
Intraoperative computed tomography for intracranial electrode implantation surgery in medically refractory epilepsy
}

\author{
Darrin J. Lee, MD, PhD, ${ }^{1}$ Marike Zwienenberg-Lee, MD, ${ }^{1}$ Masud Seyal, MD, PhD, ${ }^{2}$ \\ and Kiarash Shahlaie, MD, PhD ${ }^{1,2}$
}

Departments of ${ }^{1}$ Neurological Surgery and ${ }^{2}$ Neurology, UC Davis School of Medicine, Sacramento, California

OBJECT Accurate placement of intracranial depth and subdural electrodes is important in evaluating patients with medically refractory epilepsy for possible resection. Confirming electrode locations on postoperative CT scans does not allow for immediate replacement of malpositioned electrodes, and thus revision surgery is required in select cases. Intraoperative CT (iCT) using the Medtronic $\mathrm{O}$-arm device has been performed to detect electrode locations in deep brain stimulation surgery, but its application in epilepsy surgery has not been explored. In the present study, the authors describe their institutional experience in using the $\mathrm{O}$-arm to facilitate accurate placement of intracranial electrodes for epilepsy monitoring.

METHODS In this retrospective study, the authors evaluated consecutive patients who had undergone subdural and/or depth electrode implantation for epilepsy monitoring between November 2010 and September 2012. The 0-arm device is used to obtain iCT images, which are then merged with the preoperative planning MRI studies and reviewed by the surgical team to confirm final positioning. Minor modifications in patient positioning and operative field preparation are necessary to safely incorporate the 0 -arm device into routine intracranial electrode implantation surgery. The device does not obstruct surgeon access for bur hole or craniotomy surgery. Depth and subdural electrode locations are easily identified on iCT, which merge with MRI studies without difficulty, allowing the epilepsy surgical team to intraoperatively confirm lead locations.

RESULTS Depth and subdural electrodes were implanted in 10 consecutive patients by using routine surgical techniques together with preoperative stereotactic planning and intraoperative neuronavigation. No wound infections or other surgical complications occurred. In one patient, the hippocampal depth electrode was believed to be in a suboptimal position and was repositioned before final wound closure. Additionally, 4 strip electrodes were replaced due to suboptimal positioning. Postoperative CT scans did not differ from iCT studies in the first 3 patients in the series and thus were not obtained in the final 7 patients. Overall, operative time was extended by approximately 10-15 minutes for 0-arm positioning, less than 1 minute for image acquisition, and approximately 10 minutes for image transfer, fusion, and intraoperative analysis (total time $21-26$ minutes).

CONCLUSIONS The 0 -arm device can be easily incorporated into routine intracranial electrode implantation surgery in standard-sized operating rooms. The technique provides accurate 3D visualization of depth and subdural electrode contacts, and the intraoperative images can be easily merged with preoperative MRI studies to confirm lead positions before final wound closure. Intraoperative CT obviates the need for routine postoperative CT and has the potential to improve the accuracy of intracranial electroencephalography recordings and may reduce the necessity for revision surgery. http://thejns.org/doi/abs/10.3171/2014.9.JNS13919

KEY WORDS epilepsy; intraoperative computed tomography; image-guided surgery; electroencephalography

A PPROXIMATELY one-third of newly diagnosed epilepsy cases are refractory to medical management, and patients in these cases may be appropriate candidates for surgical intervention. ${ }^{9,15}$ The patients often undergo intracranial electroencephalography (EEG) monitoring via implanted subdural and/or depth electrodes to identify a seizure onset zone. Subdural electrode strips and grids are sometimes implanted through bur holes or

ABBREVIATIONS DBS = deep brain stimulation; EEG = electroencephalography; iCT = intraoperative CT; iMRI = intraoperative MRI.

SUBMITTED May 3, 2013. ACCEPTED September 23, 2014.

INCLUDE WHEN CITING Published online October 31, 2014; DOI: 10.3171/2014.9.JNS13919.

DISCLOSURE The authors report no conflict of interest concerning the materials or methods used in this study or the findings specified in this paper. This study was supported by the UC Davis Bronte Epilepsy Research Program. 
beyond the edges of a craniotomy; therefore, direct visualization of their final position may not be possible. Depth electrodes are often placed with a high degree of accuracy using stereotactic guidance but can occasionally be malpositioned as a result of errors in depth placement and/or lead deviation. Subdural grid and strip electrodes are more prone to suboptimal positioning since they are sometimes passed through a bur hole or beyond craniotomy edges. ${ }^{10}$ Accurate placement of invasive EEG electrodes is important for correctly identifying a seizure onset zone that may be appropriate for resection.

Preoperative MRI is typically used to evaluate for structural lesions and to plan electrode placement, and postoperative CT is often performed to confirm electrode placement. ${ }^{2,6}$ This approach is problematic, however, since the repositioning of electrodes using this strategy requires an additional operation. Intraoperative fluoroscopy can be used to confirm electrode implantation relative to a stereotactic frame, but it is not effective in frameless operations. Moreover, fluoroscopy is limited in its ability to detect medial and/or lateral lead deviations, and radiographic studies do not easily fuse with preoperative neuronavigation plans developed from CT and MRI.,11

Intraoperative CTs (iCTs) can be obtained in standard operating rooms using the portable O-arm image acquisition system (Medtronic Inc.). This technique has been described in deep brain stimulation (DBS) surgery for confirming the accurate placement of electrodes into deep brain structures,${ }^{14}$ but its use in epilepsy surgery has not been investigated. In the present study, we report our institutional experience using the $\mathrm{O}$-arm to facilitate accurate placement of intracranial electrodes for epilepsy monitoring.

\section{Methods}

The UC Davis Institutional Review Board approved this chart review. In this retrospective study, we evaluated consecutive patients who had undergone subdural and/or depth electrode implantation for epilepsy monitoring between November 2010 and September 2012.

\section{Preoperative Planning}

All patients undergo routine preoperative MRI as part of the evaluation for surgical intervention, and subsequent volumetric contrast-enhanced T1-weighted 3D MRI is performed for surgical planning. The StealthStation Cranial software package (Medtronic Inc.) is used, with the Brain Biopsy module selected for electrode planning. Surgical plans are developed for subdural electrode placement by creating a 3D rendering of the surface anatomy (Fig. 1). Depth electrode plans are developed using entry points and trajectories that meet the following criteria: 1) entering in the crest of the middle or inferior temporal gyrus, 2) avoiding transgression of a cortical sulcus, and 3) avoiding injury to visible vascular structures.

\section{O-Arm Positioning and Intraoperative Imaging}

After the induction of general anesthesia, the patient's head is secured in a Mayfield head clamp and attached to the operating table using a modified connector that will accommodate the gantry of the O-arm device. ${ }^{14}$ Patient arms are padded and tucked such that arm boards can be removed, minimizing the table width that will be needed to accommodate the diameter of the $\mathrm{O}$-arm gantry. If a standard operating table is used, the head and foot orientation is reversed to maximize the distance between the head of the bed and the base of the operating table. If a sliding table is used, it is slid to the head end of the bed to maximize this distance.

The $\mathrm{O}$-arm is then moved into position using techniques described elsewhere. ${ }^{14}$ These techniques are modified for epilepsy surgery by sliding the $\mathrm{O}$-arm below the patient's shoulders to provide maximal access to the head. The reference arc for the StealthStation navigation is positioned above the vertex of the head to provide direct line of sight to the infrared detection device without obstruction from the $\mathrm{O}$-arm gantry. Specifically, the $\mathrm{O}$-arm gantry is positioned around the torso at the level of the upper chest to allow access to create bur holes or larger craniotomies when necessary. The operative field is then prepared in a routine fashion, and a clear hip or shower curtain drape is used to cover the operative field as well as the anterior half of the O-arm machine (Fig. 2). This allows the O-arm to be moved between an operating/surgeon and an imaging/ anesthesia position without compromising the sterility of the operative field. Draping the operative field to include the $\mathrm{O}$-arm gantry requires 3 people (primary surgeon, assistant surgeon or scrub technician, and non-scrubbed circulator) and is technically simple. By allowing sufficient redundancy in drape material between the patient's head and the $\mathrm{O}$-arm ring, the machine is easily moved between the operating and imaging positions without compromising the sterility of the surgical field or colliding with the neuronavigation frame or other sterile equipment.

\section{Analysis of Intraoperative CT Scans}

After depth electrodes are placed, tunneled, and temporarily secured, the $\mathrm{O}$-arm is moved to the imaging position to acquire an $\mathrm{iCT}$ scan, which is obtained using previously described techniques that involve the entire convexity of the skull and skull base. ${ }^{14}$ We immediately transfer O-arm images to the StealthStation surgical navigation system via a direct Ethernet/Cat5 connection, and the Automerge function is used to fuse the iCT study with the preoperative 3D MRI study (Fig. 3). For successful fusion of $\mathrm{iCT}$ and MRI, studies are obtained using the 3D and Medium settings of the $\mathrm{O}$-arm, and care is taken to ensure that the entire convexity and skull base are included in the iCT study. To determine if any modifications or replacements are necessary prior to final closure, we confirm the location of subdural grid and strip electrodes on a 3D rendering of the brain surface (Fig. 1). Depth electrode entry point, trajectory, and tip location are compared with those from the preoperative plan.

\section{Results \\ Patient Population}

Ten patients underwent implantation of subdural and depth electrodes for invasive epilepsy monitoring at our Level 4 epilepsy center. The patient series included 6 wom- 


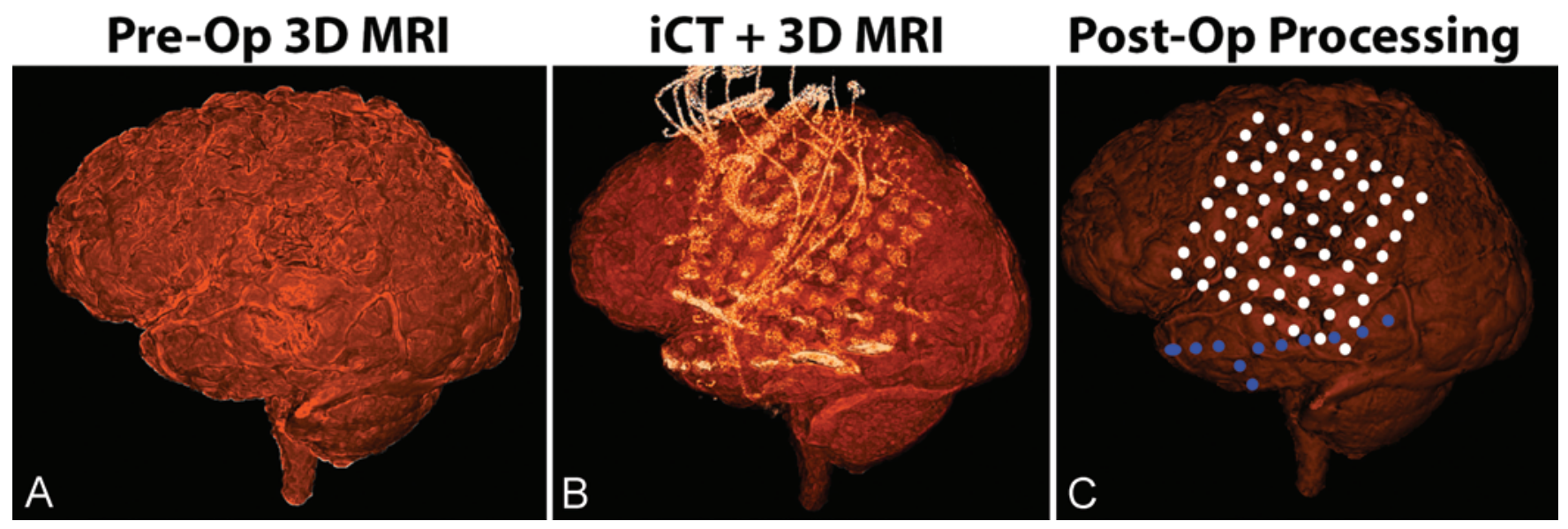

FIG. 1. Three-dimensional surface-rendering images created on a Medtronic StealthStation to guide placement of subdural grid and strip electrodes. The preoperative MR image (A) was merged with the iCT study in the operating room for immediate confirmation of subdural lead locations (B). Postoperatively, the merged image was processed to yield a 3D map of electrode locations that could be used for interpreting extraoperative EEG data (C). Blue dots represent strip electrodes, and white dots represent grid points.

en and 4 men, with an average age of 33 years. All patients underwent placement of subdural electrodes (strips and/or grids), and 8 of the 10 patients had mesial temporal lobe depth electrodes placed as well (Table 1). Four patients underwent a second-stage resection for focal epilepsy.

\section{Electrode Repositioning}

One hundred four subdural electrode arrays were placed in 10 patients using iCT confirmation; the series included 45 temporal lobe strips, 48 frontal lobe strips, and 11 grid arrays. Four strips were replaced because of suboptimal positioning, including 1 anterior temporal and 3 inferior/ventral temporal strips.

Thirty depth electrodes were placed in 8 of the 10 patients using iCT to confirm final implantation. Postoperative CT scans were also obtained in the first 3 patients in this series and were identical to the $\mathrm{iCT}$ scans with respect to electrode lead locations. One depth electrode was replaced because of suboptimal positioning of the electrode tip, which was posterior and lateral to the intended hippocampal target.

\section{Complications}

There were no surgical site infections in this clinical series. Operative time was extended by approximately 10-15 minutes for positioning the O-arm, less than 1 minute for image acquisition, and approximately $10 \mathrm{~min}$ utes for image transfer, fusion, and intraoperative analysis (total time 21-26 minutes). In cases in which electrode repositioning was performed, the operative time was prolonged approximately 12 minutes to reposition electrodes and confirm final location with a repeat iCT scan.
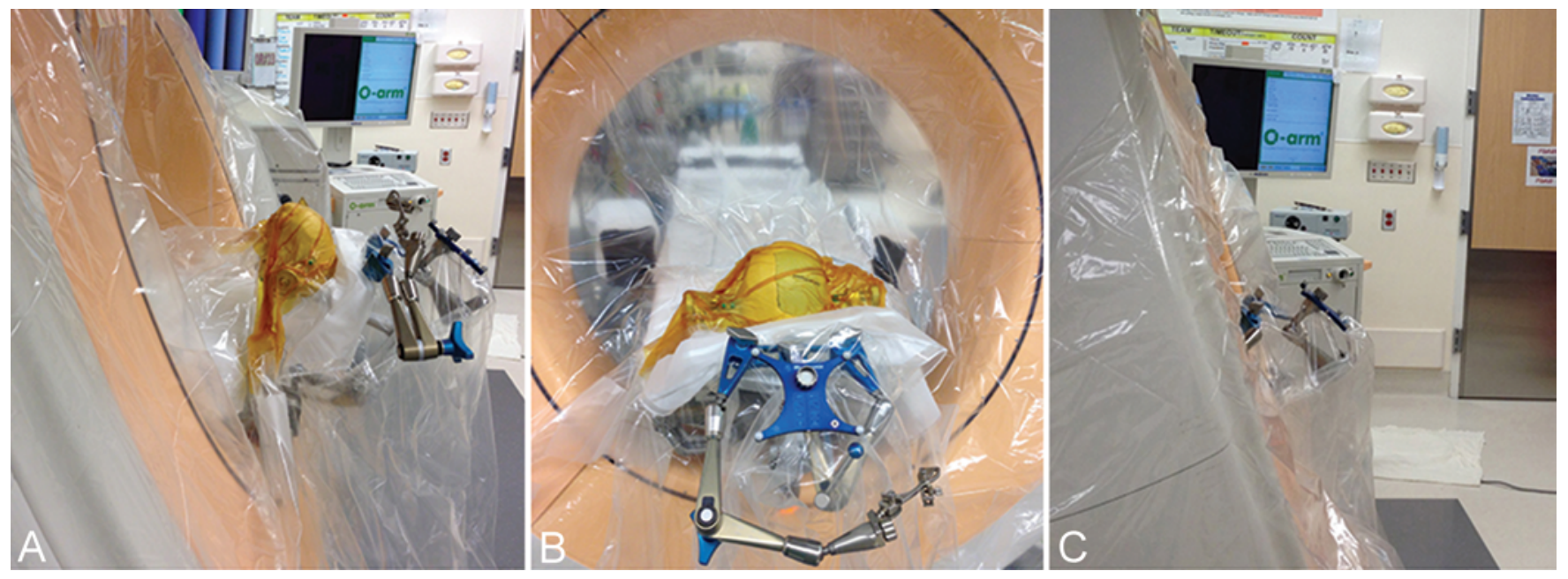

FIG. 2. Example of operating room setup of the O-arm gantry and intraoperative navigation system. In the operating/surgeon position (A), operative access is maximized, and the navigation equipment is in a midline position that does not interfere with the surgical field (B). In the imaging/anesthesia position (C), the sterility of the operative field is maintained, and navigation and stereotactic equipment are not disturbed. 

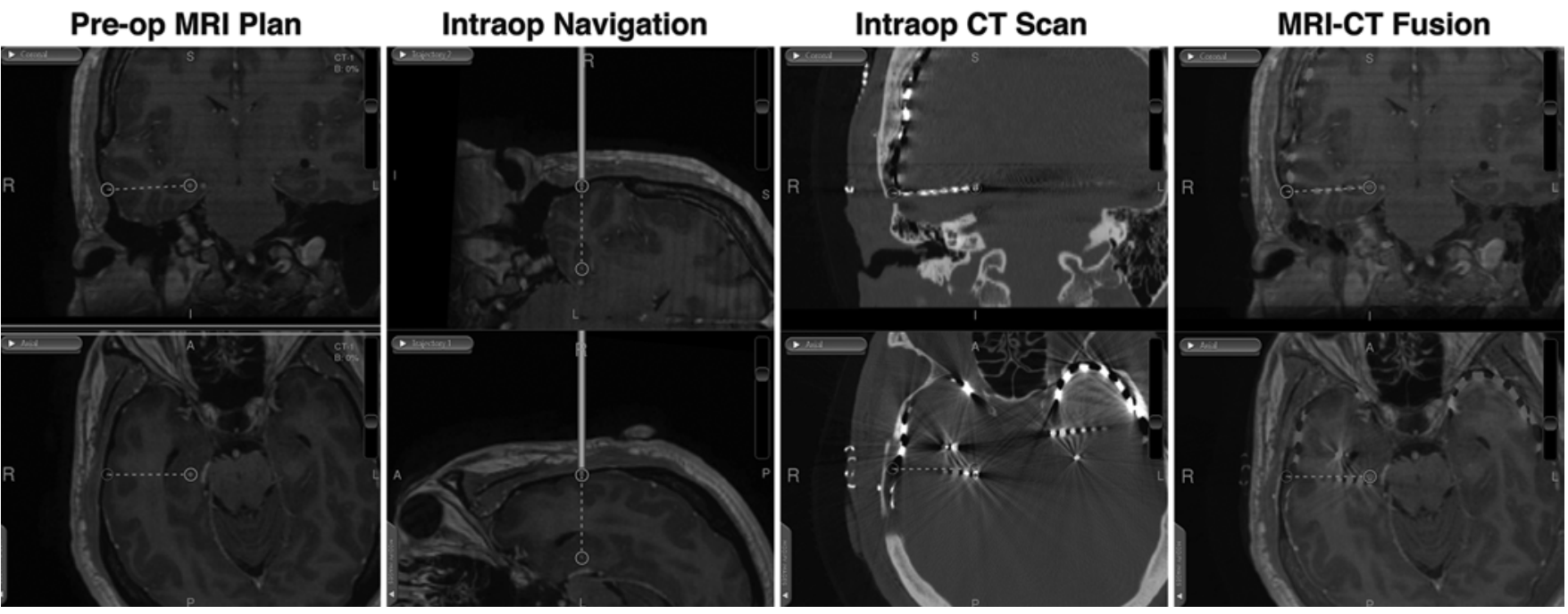

FIG. 3. Images obtained on a Medtronic StealthStation system during subdural and depth electrode placement for invasive epilepsy monitoring. Preoperative MRI was used to develop a plan to place the right hippocampal depth electrode, stereotactic guidance was used to implant the electrode along the planned trajectory, and an intraoperative CT scan was obtained and then merged with the preoperative MRI study to confirm trajectory and depth as well as the final anatomical location of the electrode.

\section{Discussion}

In this study, we demonstrated the feasibility of incorporating the O-arm machine into routine epilepsy surgery to facilitate the accurate placement of intracranial recording electrodes. Simple technical modifications allow the machine to be positioned such that it does not interfere with surgical access to the patient for bur hole or craniotomy procedures and can be easily moved between operating and imaging positions while maintaining the integrity of the sterile field. Intraoperative CT easily merges with preoperative planning studies to provide accurate 3D confirmation of subdural and depth electrode placement, allowing any necessary positioning adjustments to be made before leaving the operating room. This technique has the potential to improve the accuracy and quality of invasive EEG data and reduce the need for reoperation to replace electrodes.
The ability to obtain 3D imaging data during neurosurgical procedures has many potential benefits and has been a topic of interest for the past few decades. Intraoperative MRI (iMRI) can provide excellent visualization of the brain anatomy, but it can only be performed in dedicated operative MRI suites or with smaller iMRI devices that have more limited imaging capabilities. Because of the ferromagnetic qualities of standard invasive electrodes and the intraoperative equipment needed to interrogate them during surgery, iMRI is not a feasible option for 3D imaging during invasive EEG electrode implantation.

Intraoperative 3D imaging has been described in a variety of neurosurgical applications, including $\mathrm{CT}$ for brain tumor or skull base biopsy3,8 and 3D fluoroscopy for different spine operations. ${ }^{7,12,13,17}$ Over the past few years, interest in using intraoperative $\mathrm{CT}$ scanning to confirm placement of DBS electrodes has been increasing. The Medtronic

TABLE 1. Electrode implantation patient series

\begin{tabular}{|c|c|c|c|c|c|c|c|c|}
\hline \multirow[b]{2}{*}{$\begin{array}{l}\text { Patient Age } \\
\text { (yrs), Sex }\end{array}$} & \multicolumn{4}{|c|}{ Right Electrodes } & \multicolumn{4}{|c|}{ Left Electrodes } \\
\hline & $\begin{array}{l}\text { Frontal } \\
\text { Strips }\end{array}$ & $\begin{array}{c}\text { Temporal } \\
\text { Strips }\end{array}$ & $\begin{array}{c}\text { Temporal } \\
\text { Depths }\end{array}$ & Grids & $\begin{array}{c}\text { Frontal } \\
\text { Strips }\end{array}$ & $\begin{array}{c}\text { Temporal } \\
\text { Strips }\end{array}$ & $\begin{array}{c}\text { Temporal } \\
\text { Depths }\end{array}$ & Grids \\
\hline $40, \mathrm{~F}$ & 0 & 3 & 2 & 0 & 3 & 3 & 2 & 1 \\
\hline $32, F$ & 3 & 3 & 2 & 0 & 3 & 3 & 2 & 0 \\
\hline $20, M$ & 3 & 3 & 2 & 0 & 3 & 3 & 2 & 0 \\
\hline $38, F$ & 3 & 3 & 2 & 0 & 3 & 3 & 2 & 0 \\
\hline $17, \mathrm{M}$ & 3 & 3 & 2 & 0 & 3 & 3 & 2 & 4 \\
\hline $17, \mathrm{M}$ & 3 & 0 & 0 & 0 & 3 & 3 & 2 & 2 \\
\hline $49, \mathrm{~F}$ & 0 & 3 & 2 & 0 & 0 & 3 & 2 & 0 \\
\hline $38, M$ & 3 & 0 & 0 & 1 & 6 & 0 & 0 & 0 \\
\hline $56, F$ & 0 & 3 & 2 & 0 & 0 & 3 & 2 & 0 \\
\hline $27, \mathrm{~F}$ & 3 & 0 & 0 & 3 & 3 & 0 & 0 & 0 \\
\hline Total & 21 & 21 & 14 & 4 & 27 & 24 & 16 & 7 \\
\hline
\end{tabular}


$\mathrm{O}$-arm is a portable device that is available at most institutions given its use in spine surgery. The $\mathrm{O}$-arm can be easily incorporated into standard frame-based ${ }^{1,5,14}$ and frameless ${ }^{16}$ DBS surgery with simple technical modifications. This imaging technique has been shown to provide accurate depictions of lead locations and has the potential to reduce the number of malpositioned DBS electrodes that might require subsequent revision surgery..$^{14}$

In the present study, we confirmed that these advantages of iCT extend to epilepsy surgery. The techniques used to incorporate the O-arm device are simple to apply in standard-sized operating rooms and do not interfere with the ability to safely perform bilateral bur hole or craniotomy procedures to implant electrodes. Furthermore, direct line of sight can be maintained between the infrared detection device of neuronavigation systems and the aiming guides used for depth electrode implantation, so that current surgical procedures need not be modified. Computed tomography images can be obtained at any stage of the operation and can be used to confirm the location of depth and surface electrodes using preexisting software packages that rapidly merge iCT with preoperative planning MRI studies. The technique has many potential advantages: 1) more useful intraoperative diagnostic EEG data given accurate placement of electrode contacts, 2) ability to reposition malpositioned electrodes before closing the surgical wound, 3) decreased hospital stay because of a reduced reoperation rate, and 4) no need for routine postoperative CT scanning to confirm electrode positions, reducing the need for patient transport after electrode implantation.

The potential drawbacks to this technique warrant consideration. First, the $\mathrm{O}$-arm provides excellent visualization of bone and implanted hardware (for example, electrode contacts), but it is inadequate for soft-tissue imaging. As a result, it cannot be used to reliably identify the presence of an intracranial hematoma, and the location of an electrode relative to deep brain structures can only be deduced by fusing O-arm images with preoperative MRI studies. If there is significant concern regarding intracerebral hematoma from the operation, a routine postoperative CT scan will still be necessary. In facilities with an operative CT suite (rather than a portable device), the intraoperative scan could provide adequate imaging of soft tissue and/or a hematoma as well as the electrode contacts.

Second, the O-arm is large, and careful planning is required to maintain field sterility and provide adequate surgical access. We did not have any wound infections or other intraoperative complications associated with the presence of the $\mathrm{O}$-arm machine during surgery, but it is important to note that our surgical team had significant experience using the $\mathrm{O}$-arm during DBS surgery and other intracranial neurosurgical procedures before we incorporated the device into our surgical epilepsy practice. Moreover, repeat imaging with the $\mathrm{O}$-arm introduces the possibility of increased patient exposure to ionizing radiation, particularly if electrodes are repositioned and an additional iCT scan is obtained. Zhang et al. have characterized $\mathrm{O}$-arm dosimetry ${ }^{18}$ and have shown that when the $\mathrm{O}$-arm is set at the $3 \mathrm{D}$ imaging mode it delivers $50 \%$ of the radiation dose of a 64-slice CT scanner. Thus, for patients who do not require repeat intraoperative or postoperative imaging, the $\mathrm{O}$-arm has the potential to reduce total radiation exposure that would otherwise occur with routine postoperative CT scans. Finally, if an O-arm device is not already available at a particular institution, there is a significant capital cost associated with purchasing the device that could increase the costs of iCT-assisted intracranial electrode implantation surgery.

\section{Conclusions}

The $\mathrm{O}$-arm device can be easily incorporated into routine electrode implantation surgery with minimal effects on operative time. By merging $\mathrm{iCT}$ images with preoperative MRI studies, the surgical team can visually confirm subdural and depth electrode positions before final wound closure. This approach improves the accuracy of intracranial electrode placement and may reduce the necessity for revision surgery.

\section{References}

1. Caire F, Gantois C, Torny F, Ranoux D, Maubon A, Moreau JJ: Intraoperative use of the Medtronic O-arm for deep brain stimulation procedures. Stereotact Funct Neurosurg 88:109-114, 2010

2. Chamoun RB, Nayar VV, Yoshor D: Neuronavigation applied to epilepsy monitoring with subdural electrodes. Neurosurg Focus 25(3):E21, 2008

3. Engle DJ, Lunsford LD: Brain tumor resection guided by intraoperative computed tomography. J Neurooncol 4:361370, 1987

4. Erõss L, Bagó AG, Entz L, Fabó D, Halász P, Balogh A, et al: Neuronavigation and fluoroscopy-assisted subdural strip electrode positioning: a simple method to increase intraoperative accuracy of strip localization in epilepsy surgery. Technical note. J Neurosurg 110:327-331, 2009

5. Fiegele T, Feuchtner G, Sohm F, Bauer R, Anton JV, Gotwald T, et al: Accuracy of stereotactic electrode placement in deep brain stimulation by intraoperative computed tomography. Parkinsonism Relat Disord 14:595-599, 2008

6. Hogan RE, Lowe VJ, Bucholz RD: Triple-technique (MR imaging, single-photon emission CT, and CT) coregistration for image-guided surgical evaluation of patients with intractable epilepsy. AJNR Am J Neuroradiol 20:1054-1058, 1999

7. Ito Y, Sugimoto Y, Tomioka M, Hasegawa Y, Nakago K, Yagata Y: Clinical accuracy of 3D fluoroscopy-assisted cervical pedicle screw insertion. Clinical article. J Neurosurg Spine 9:450-453, 2008

8. Kitai R, Sato K, Ido K, Sakuma T, Handa Y, Kubota T: Single burr hole surgery for the spheno-orbital fibrous dysplasia using intraoperative computed tomography. Minim Invasive Neurosurg 48:44-46, 2005

9. Kwan P, Sander JW: The natural history of epilepsy: an epidemiological view. J Neurol Neurosurg Psychiatry 75:1376-1381, 2004

10. LaViolette PS, Rand SD, Ellingson BM, Raghavan M, Lew SM, Schmainda KM, et al: 3D visualization of subdural electrode shift as measured at craniotomy reopening. Epilepsy Res 94:102-109, 2011

11. Miller KJ, Makeig S, Hebb AO, Rao RPN, denNijs M, Ojemann JG: Cortical electrode localization from X-rays and simple mapping for electrocorticographic research: the "Location on Cortex" (LOC) package for MATLAB. J Neurosci Methods 162:303-308, 2007 
12. Nottmeier EW, Seemer W, Young PM: Placement of thoracolumbar pedicle screws using three-dimensional image guidance: experience in a large patient cohort. Clinical article. J Neurosurg Spine 10:33-39, 2009

13. Rajasekaran S, Vidyadhara S, Shetty AP: Intra-operative IsoC3D navigation for pedicle screw instrumentation of hangman's fracture: a case report. J Orthop Surg (Hong Kong) 15:73-77, 2007

14. Shahlaie K, Larson PS, Starr PA: Intraoperative computed tomography for deep brain stimulation surgery: technique and accuracy assessment. Neurosurgery 68 (1 Suppl Operative):114-124, 2011

15. Sillanpää M, Schmidt D: Natural history of treated childhood-onset epilepsy: prospective, long-term population-based study. Brain 129:617-624, 2006

16. Smith AP, Bakay RA: Frameless deep brain stimulation using intraoperative $\mathrm{O}$-arm technology. Clinical article. J Neurosurg 115:301-309, 2011

17. Villavicencio AT, Burneikiene S, Bulsara KR, Thramann JJ: Intraoperative three-dimensional fluoroscopy-based computerized tomography guidance for percutaneous kyphoplasty. Neurosurg Focus 18(3):E3, 2005
18. Zhang J, Weir V, Fajardo L, Lin J, Hsiung H, Ritenour ER: Dosimetric characterization of a cone-beam O-arm imaging system. J XRay Sci Technol 17:305-317, 2009

\section{Author Contributions}

Conception and design: Shahlaie, Zwienenberg-Lee. Acquisition of data: Shahlaie, Lee, Seyal. Analysis and interpretation of data: Shahlaie, Lee, Zwienenberg-Lee. Drafting the article: Lee. Critically revising the article: all authors. Reviewed submitted version of manuscript: all authors. Approved the final version of the manuscript on behalf of all authors: Shahlaie. Study supervision: Shahlaie.

\section{Correspondence}

Kiarash Shahlaie, Department of Neurological Surgery,

University of California, Davis School of Medicine, 4860 Y St., Ste.3740, Sacramento, CA 95817. email:krshahlaie@ucdavis. edu. 